\title{
EL PENSAMIENTO DE CARLOS GAVIRIA DÍAZ A PROPÓSITO DE LA DOSIS PERSONAL: ENTRE EL LIBRE DESARROLLO A LA PERSONALIDAD Y EL DERECHO A LA SALUD
}

Carlos Fernando Amaya Rodríguez Recibido: Noviembre 30 de 2016 Aprobado: Diciembre 15 de 2016

\section{RESUMEN}

La presente investigación se fundamenta en una revisión del progresismo del jurista colombiano Carlos Gaviria Díaz (Q.E.P.D.), respecto de su pensamiento jurídico sobre determinados temas etiquetados como casos difíciles o tabú, en donde se ve un marcado avance en la solución de conflictos suscitados por el choque entre principios y derechos del orden fundamental, para ello el proyecto se propone analizar diferentes sentencias en la que el citado jurista plantea un cambio en la compresión de estos asuntos, de tal suerte que fijará la línea que sobre los mismos mantendrá la Corte Constitucional Colombiana desde sus inicios en 1991, respecto del reconocimiento y protección de derechos. Así las cosas en el presente artículo se abordará la temática de la dosis personal como punto de choque y convergencia entre el libre desarrollo de la personalidad y el derecho a la salud (entendido este como un deber del Estado Colombiano para con sus asociados).

Palabras clave: Carlos Gaviria Díaz, Corte Constitucional, Ponderación, Dosis Personal, Libre desarrollo de la personalidad, Derecho a la salud.

\footnotetext{
* El presente artículo es un avance de proyecto de investigación en el marco del proyecto titulado El Pensamiento jurídico de Carlos Gaviria Díaz en los casos difíciles de la Corte Constitucional, desarrollado dentro de la línea Estado, Derecho y Sociedad del Grupo de Investigaciones Socio Jurídicas UNICOC GISU. ** Abogado de UNICOC, Especialista en Contratación Estatal de la Universidad de La Sabana y Doctorando de la Universidad de Buenos Aires. Actualmente se desempeña como profesor de apoyo a la investigación del Colegio Jurídico y de Ciencias Sociales de La Institución Universitaria Colegios de Colombia UNICOC. Así mismo es miembro adjunto extranjero de la Asociación Argentina de Justicia Constitucional. En la investigación es miembro del Grupo de Investigaciones Socio Jurídica de UNICOC GISU y desarrolla su trabajo académico en la línea de Derecho, Estado y Sociedad. Correo electrónico: cfamaya@unicoc.edu.co
} 
THE THOUGHT OF CARLOS GAVIRIA DIAZ ABOUT THE PERSONAL

DOSE: BETWEEN THE FREE DEVELOPMENT OF PERSONALITY AND THE RIGHT TO HEALTH

\begin{abstract}
This research is based on a review of progressivism colombian jurist Carlos Gaviria Diaz (R.I.P), regarding his legal thinking on certain issues labeled as difficult cases or taboo, where one sees a marked advance in the solution of conflicts raised from the collision between principles and fundamental rights. For this purpose, the project aims to analyze different sentences in which the jurist quoted proposes a change in the understanding of these issues, in such a way as to establish the background that the colombian Constitutional Court will maintain from its beginnings in 1991 in the recognition and protection of rights. Thus, the present article will address the issue of personal dose as a point of collision and convergence between the free development of personality and the right to health (understood as a duty of the Colombian State to its associates).
\end{abstract}

KEYWORDS: Carlos Gaviria Díaz, Colombia Constitutional Court, Weighting, Personal Dose, Free Development of personality, Right to health.

\title{
O PENSAMENTO DE CARLOS GAVIRIA DÍAZ REFERENTE À DOSE PESSOAL: ENTRE O LIVRE DESENVOLVIMENTO DA PERSONALIDADE E O DIREITO À SAÚDE
}

\section{RESUMO}

A presente pesquisa é baseada em uma revisão do progressismo jurista colombiano Carlos Gaviria Díaz (qepd), respeito ao seu pensamento jurídico em certas questões identificadas como casos difíceis ou tabu, onde há um progresso acentuado na resolução de conflitos decorrentes da choque entre princípios e direitos de ordem fundamental, para o qual o projeto é analisar diferentes sentenças em que este jurista levanta uma mudança na compreensão destas questões, de tal forma que irá definir a linha a manter no Tribunal Constitucional da Colômbia desde a sua criação em 1991, respeito ao reconhecimento e protecção dos direitos. É assim que neste trabalho se apresentará a questão da dose pessoal como um ponto de choque e convergência entre o livre desenvolvimento da personalidade e do direito à saúde (entendido este como um dever do Estado colombiano para com os seus associados). 
Palavras chaves: Carlos Gaviria Díaz, Tribunal Constitucional, ponderação, dose pessoal, livre desenvolvimento da personalidade, direito à saúde.

\section{INTRODUCCIÓN}

Los derechos como atributo de la esencia humana resultan en un escenario de especial interés para el investigador jurídico. Lo anterior en virtud al alto número de tensiones y problemas jurídicos que se gestan en su alrededor. Esta situación genera la necesidad de diseñar instrumentos y modelos para resolver las problemáticas que se presentan cuando hay conflicto entre los derechos, vulneración por parte de los estados a estos o entre personas naturales, entre otros ejemplos.

En ese marco los Estados han diseñado desde sus textos constitucionales Cortes o Tribuales destinados a dirimir o limar esas tensiones, así como garantizar la protección y goce de los derechos de las personas. Así pues, Colombia en el año de 1991 en su texto constitucional procede a la creación de un Alto Tribunal destinado a cumplir con las funciones antes expuestas, dicho Tribunal se denomina Corte Constitucional, el cual, además se le da la tarea de ser el guardián de la supremacía de los preceptos, principios y esencia de la Carta Política Colombiana.

Por esta Corte se ha discutido y ventilado una infinidad de asuntos relevantes a los derechos humanos reconocidos o no por el texto constitucional colombiano. Buscando en todo caso propender por la optimización del derecho como instrumento para la vida en sociedad, temas como el aborto, el matrimonio igualitario, la dosis personal, el derecho a la salud de los colombianos, la educación, la igualdad, la dignidad humana entre muchos otros, han sido analizados por los magistrados de esta Corte a la luz de las normas constitucionales colombianas y de los instrumentos del orden internacional que por medio del bloque de constitucionalidad hacen parte del orden jurídico interno con el mismo rango o jerarquía que las disposiciones del texto supremo, así como también a la luz de los métodos de interpretación tradicionales y contemporáneos de las ciencias jurídicas.

Al inicio de dicho tribunal se contó con la presencia de uno de los juristas con mayor relevancia en los últimos años en Colombia, el abogado Carlos Gaviria Díaz, quien por medio de sus ponencias respecto de los casos asignado a su despacho, representó una nueva forma de 
comprender la dinámicas jurídicas del enfrentamiento de derechos y solución de casos tabúes, de manera adelantada a la visión altamente conservadora que, durante muchos año, imperó e impera en la sociedad colombiana.

En el presente documento se presenta una de las sentencias que con mayor facilidad se recuerda del citado Magistrado, en donde se esboza con claridad la visión que desde su pensamiento se debe atender para la solución de las controversias ya referidas, así como el tratamiento a los temas complejos y tabúes de la sociedad, para ello en un primer momento se presentan brevemente los hechos que dan lugar al pronunciamiento del Alto Tribunal Constitucional, las consideraciones que lo llevan a tomar su decisión para, finalmente, exponer una reflexión sobre el mismo.

El presente trabajo toma por su naturaleza la metodología de investigación de tipo descriptivo, ya que con ella se busca la descripción de las situaciones jurídicas que son objeto de la investigación, esto es, la solución y visión que desde el pensamiento académico de Carlos Gaviria Díaz se da a los denominados conflictos o choque de principios y derechos fundamentales, que coloquialmente han sido denominados por diferentes agentes sociales como temas tabúes. De igual forma, la investigación en general propone una exploración en la jurisprudencia de la Corte Constitucional colombiana en donde el magistrado ponente haya sido el referido jurista, con el fin de generar líneas de interpretación constitucional desde su visión sobre los temas en comento. Para cada sentencia se hará un revisión de los hechos o los cargos formulados contra las normas acusadas, una revisión de las consideraciones de la Corte y finalmente una reflexión desde la doctrina en torno a ella.

\section{LA SENTENCIA C-221 DE 1.994: PLANTEO DE LOS CARGOS CONTRA LA LEY 30 DE 1.986.}

En el año de 1.994 la Corte Constitucional procede a pronunciarse respecto del tema de la dosis personal, la cual previo a la sentencia se encontraba penalizada por las normas punitivas del Estado colombiano, situación que a juicio del actor de la demanda limitaba el libre desarrollo de la personalidad de los colombianos, bajo los argumentos de la salubridad pública y la limitación a la autonomía de las personas en lo que se refiere a su propio cuerpo y vida. No debe en todo caso perderse de vista la importancia que la posición de garante que tiene el Estado en la protección de la salud de sus asociados. 
En concreto el accionante, por medio de la acción pública de inconstitucionalidad demanda a la Corte que declare inexequibles el literal j) del artículo $2^{\circ}$. y el artículo 51 de la ley 30 de 1986, al formular los siguientes cargos:

A. Las normas en comento transgreden los límites del Estado en la intervención de la salud personal, en el sentido de que el Estado no puede garantizar la recuperación plena de un enfermo, así en el caso del drogadicto, no puede privarle de la sustancia que afecta su salud en primer lugar psíquica y, por consecuente, física, la cual en este caso es la sustancia estupefaciente de la cual depende; si lo hiciere estaría infringiendo el art. 366 de la carta política de 1.991 afirmando en palabras del demandante que

Si el Estado no puede garantizar la recuperación de la salud de los enfermos psicofisiológicos de drogadicción o toxicomanía, porque no existe clínicamente el tratamiento radical y científico que asegure su curación, tampoco puede el Estado impedir o limitar el uso del medicamento que le procura alivio al sufrimiento del enfermo. Los estupefacientes son parte integral de la enfermedad de drogadicción o toxicomanía y a la vez, son el medicamento que alivia el dolor y el sufrimiento de los enfermos incurables. (Corte Constitucional, Sentencia C-221-94, Magistrado Ponente Carlos Gaviria Díaz, Expediente N ${ }^{\circ}$. D429),

Dentro del mismo cargo indica que el Estado no puede privar de la libertad de las personas que son drogadictas o cualquier otra afección psicofisiológica, pues se estarían vulnerando los mandatos del texto constitucional contenidos en los artículos 5, 28, 29, 34 y 49 de la Carta; finalmente y en relación a este cargo se decanta a exponer que sancionar punitivamente a un drogadicto por el consumo de estupefacientes y porte de la dosis personal vulnera los artículos 28 y 91 de la Constitución, ya que el poder punitivo del Estado solo es para las transgresiones del ordenamiento jurídico que, a juicio del accionante, afecten a la sociedad y los bienes jurídico tutelados y mal hace el Estado en sancionar a la persona que se perjudica a sí misma.

B. Como un segundo cargo frente a las normas acusadas, se presenta la discriminación para con los drogadictos. Lo anterior con base a la distinción entre estos y los enfermos incurables que requieren tratamiento por medio de drogas que generan adicción (como la morfina, 
un derivado de la heroína), y más evidente aún entre la distinción que se hace entre los dependientes del alcohol y la nicotina (sustancias que son también estupefacientes y estimulantes del sistema nervioso) a quien se les otorga la concesión de consumidores de drogas socialmente aceptadas, mientras que a los demás se les etiqueta como delincuentes, situación reconocida en el marco de la misma Ley 30 de 1986, profundizándose aún más la discriminación al hablar entre drogadictos más o menos enfermos, afirma el accionante que

El nicotinómano y el alcohólico son tan drogadictos y toxicómanos como el marihuanero y el cocainómano; pero, se incurre en trato discriminatorio cuando se da a los dos primeros el tratamiento legal de adictos socialmente aceptados, mientras se trata a los demás consumidores de drogas como contraventores o delincuentes, dependiendo de qué tan enfermos estén. (Corte Constitucional, Sentencia C-221-94, Magistrado Ponente Carlos Gaviria Díaz, Expediente Nº. D-429).

C. Finalmente expone en un tercer cargo el tratamiento médico que la ley objeto de discusión prevé para los drogadictos, pues en principio nadie puede limitar y definir si desea o no estar enfermo; en segundo lugar en el evento de ser objeto de la internación en un centro siquiátrico hasta su recuperación, se generaría una pena de suerte imprescriptible en la eventualidad de no obtener una recuperación de la dependencia psicofisiológica de la sustancia de la cual depende el drogadicto, vulnerándose así los artículos 28 y 34 de la Constitución, así como la regla que la discusión y decisión sobre la libertad o no de una persona no compete al médico tratante, sino exclusivamente a un Juez de la República de Colombia. Finalmente reafirma su cargo bajo el argumento de que el Estado si desea hacer una política de tratamiento médico para los drogadictos, esto se debe hacer en el marco de las infraestructuras adecuadas y no en centros anexos a las cárceles y muchos menos en "tugurios infrahumanos donde se violan los derechos humanos de los ENFERMOS." (Corte Constitucional, Sentencia C-22194, Magistrado Ponente Carlos Gaviria Díaz, Expediente Nº. D-429)

De los cargos planteados debe advertirse el insistente argumento del accionante de reconocer al drogadicto no como un delincuente, sino, por el contrario, una persona enferma e incurable que debe recibir un tratamiento médico especial por parte del Estado, en condiciones dignas que le permitan sobrellevar su vida sin aflicciones mayores a las que ya su dependencia genera en un absoluto respeto por el libre desarrollo de la 
personalidad como expresión de la autonomía de la que gozan todos los colombianos.

Así mismo, se tiene presente el choque entre la necesidad del Estado de garantizar una política pública en salud adecuada para el tratamiento de estas personas, el derecho al libre desarrollo de la personalidad y la igualdad entre los consumidores o no de sustancias psicoactivas. Colisión que deberá ser limada y resuelta por parte de la Corte Constitucional Colombiana con base en los argumentos y consideraciones que se expondrán en el apartado que sigue.

\section{LOS ARGUMENTOS Y CONSIDERACIONES DE LA CORTE CONSTITUCIONAL.}

Bajo los cargos formulados contra la Ley 30 de 1.986 en la demanda objeto de decisión, debe la Corte Constitucional revolver si los preceptos de la norma jurídica en comento vulneran los límites del Estado en la intervención en la salud de las personas, de igual forma se presenta como fuente de una eventual discriminación para con los drogadictos al clasificarles bajo la etiqueta de delincuente y no enfermo y finalmente sí puede el médico tratante generar una detención indefinida de una persona hasta no recuperarse de su dependencia a las sustancias alucinógenas.

Así mismo, se hace presente un conflicto entre los derechos a la salud y al libre desarrollo de la personalidad, toda vez que la norma que se acusa de inexequible se justifica en la necesidad de mantener una política de salud pública en el tratamiento de las personas que consumen sustancias psicoactivas en el sentido de obligar al drogadicto a tomar un tratamiento a fin de recuperarse de su afección apuntando a ser un ciudadano nuevamente útil y en perfectas condiciones de salud.

En primer lugar, la Corte procede a analizar el cargo formulado a las sanciones que la ley impone a quien porte y tenga consigo dosis personal de las sustancias relacionadas en literal j del artículo 2 de la Ley 30 1986, a fin de determinar si el Estado por medio del poder legislativo puede o no limitar el comportamiento de las personas. Afirmado que si bien el poder legislativo puede establecer normas de conducta de las personas en sociedad, es atrevido e infringe el desarrollo de la personalidad al dictaminar cómo debe comportarse para consigo mismo la persona, en la medida de que su conducta no atenta contra la órbita y desarrollo de la personalidad de los otros. 
Sin embargo, hace referencia al mandato constitucional en el artículo 49 del texto superior al afirmar que toda persona "tiene el deber de procurar el cuidado integral de su salud y la de su comunidad." La anterior referencia es realizada con el fin de proponer tres posibilidades de interpretación sobre el tema, las cuales me permito citar a continuación:

1). Se trata de un mero deseo del Constituyente, llamado a producir efectos psicológicos que se juzgan plausibles, pero en modo alguno generador de un deber jurídico genérico, susceptible de plasmarse en la tipificación de una conducta penal.

2). El Estado colombiano se asume (en tanto que sujeto pretensor) dueño y señor de la vida de cada una de las personas cuya conducta rige y, por eso, arrogándose el papel de Dios, en la concepción teológica, prescribe, más allá de la órbita del derecho, comportamientos que sólo al individuo atañen y sobre los cuales cada persona es dueña de decidir.

3). Toma en consideración las consecuencias, frente a otros, de la conducta individual y por esa razón la hace objeto de regulación jurídica, v.gr.: la situación de desamparo en que puede quedar la familia del drogadicto; la privación a la comunidad de una persona potencialmente útil; el peligro que para los demás puede entrañar la conducta agresiva desatada por el consumo de las sustancias indicadas en la ley. (Corte Constitucional, Sentencia C-221-94, Magistrado Ponente Carlos Gaviria Díaz, Expediente $\mathrm{N}^{\circ}$. D-429)

De las posibilidades de interpretación del mandato constitucional ya referenciado y del cual pareciere que la ley objeto de análisis por el tribunal y solo a juicio de la Corte prospera es la primera de ellas. En el sentido de exponer que el mandato constitucional es solo un deseo del Constituyente, que debe ser o no aceptado por el legítimo titular de la salud, quien no es otro que cada individuo amparado en el régimen constitucional colombiano, así mismo las prohibiciones y sus limitaciones o sanciones solo se pueden ejercer cuando con el comportamiento se afecte la órbita de los derechos de los demás ${ }^{1}$ lo anterior y en palabras de la Corte "la Carta Política actual y la filosofía liberal que la inspira, determinante de que sólo las conductas que interfieran con la órbita de la libertad y los intereses ajenos, pueden ser

1 Es evidente la relación y referencia con una de las reglas básicas del derecho la cual se exponen como "el límite de mis derechos es donde inician los derechos de los demás". 
jurídicamente exigibles." (Corte Constitucional, Sentencia C-221-94, Magistrado Ponente Carlos Gaviria Díaz, Expediente $N^{\circ}$. D-429) Y el estar enfermo ciertamente no se encuadra desde la sana lógica en el postulado previamente expuesto.

Con base en lo anterior está llamado a prosperar el cargo formulado con relación a la vulneración de los limites del poder del Estado a interferir en el desarrollo de las personas, incluso cuando se habla de temas como los relacionados con el estado de Salud, pues si bien la Constitución de 1.991 expresó que es un deber de cada uno de los colombianos preservar su buena salud, esto debe ser entendido como un deseo que el Constituyente vio como bueno, mas no como una autorización al poder legislativo a castigar a quien no desee o entienda como bueno el precepto de gozar de buena salud.

Así mismo, se hace evidente la apreciación de enfermo que hace la Corte sobre el drogadicto, desvirtuando al parecer la concepción de delincuente que sobre esta etiqueta se deposita, lo anterior como resultado del estudio de las otras dos posibilidades hermenéuticas o de interpretación respecto al articulo 49 de la Constitución Política de 1991. Esta distinción será de utilidad para poder hacer el análisis de los otros dos cargos presentados en la demanda.

En armonía con lo anterior, la Corte procede a revisar el cargo formulado frente a la obligatoriedad de recibir tratamiento médico de parte las personas con dependencia de una sustancia alucinógena o estupefaciente, entendiéndose este tratamiento como la sanción imponible a quien consuma este tipo de sustancias. Frente a esta dicotomía hermenéutica de la norma en comento, la Corte indica que de entrada la misma adolece de todo respeto por la Constitución Política, pues no se puede sancionar a nadie (penal ni administrativamente) cuando no presenta un riesgo o amenaza a la sociedad, tal como es el caso de los drogadictos ya que el daño es autocausado a ellos mismos por la dependencia de la sustancia alucinógena de la cual depende "su bienestar psicofisiológico." Afectándose de este modo el libre desarrollo de la personalidad, pues el Estado pretendiendo tener el control total de la vida de sus ciudadanos impone obligatoriamente tomar el tratamiento, situación que a juicio de la Corte es un ejemplo de las políticas totalitarias y eugenésicas de los Estados para excluir de la sociedad a las personas que son defectuosas, por así decirlo, situación que no es compatible en ningún aspecto con el Estado Social de Derecho en el cual se construye Colombia. 
Por los motivos anteriores la Corte encuentra discriminatoria la imposición de la medida de tomar un tratamiento medico y que este tratamiento sea la internación en un centro especializado en atención a personas con dependencia de alucinógenos, la cual ha de durar hasta que se recupere la salud del afectado, así como en la limitación del derecho al libre desarrollo de la personalidad bajo los argumentos anteriormente expuestos. Por todo lo anterior se advierte que el cargo enunciado ha de prosperar y por ende la norma es declarada inexequible.

Frente al cargo de la limitación del derecho al libre desarrollo de la personalidad con ocasión de sancionar a quien consuma estupefacientes con la internación en un centro médico a fin de recibir tratamiento, la Corte procede a revisar el artículo 16 de la Constitución Política de 1.991 el cual establece que el derecho al libre desarrollo de la personalidad de cada uno de los colombianos no tendrá más limitaciones de aquellas que surgen de los derechos de los demás y el orden jurídico.

Bajo dicho paralelo la Corte advierte que el legislador no puede establecer más limitaciones que las se encuentren en armonía con el espíritu de la Constitución, así mismo el consagrar el libre desarrollo de la personalidad como un derecho, el Constituyente reconoció la autonomía de las personas para el desarrollo de su vida o en palabras de la propia Corte el principio implica "un fin en sí misma y no un medio para un fin, con capacidad plena de decidir sobre sus propios actos $\mathrm{y}$, ante todo, sobre su propio destino." (Corte Constitucional, Sentencia C221-94, Magistrado Ponente Carlos Gaviria Díaz, Expediente N. D429) mal se haría ahora que al reconocer la autonomía de las personas sea el Estado quien les diga que esa autonomía sea hacer lo bueno, y el mismo Estado sea quien defina qué se debe entender por bueno.

Entonces se tiene claro que la autonomía y libertad de las personas sobre su vida solo puede ser limitada por los derechos de los demás y las normas jurídicas coherentes con el espíritu de la Constitución. Así pues, si la decisión autónoma del drogadicto de consumir las sustancias solo afecta su esfera personal y por consecuente no encuentra cabida en el marco de la Constitución la limitación del desarrollo de la personalidad, permitiendo así que bajo dichas consideraciones la Corte estime que el cargo está llamado a prosperar $\mathrm{y}$, por ende, se procede a declarar la inexequibilidad de la norma acusada con base en dicho argumento.

Después de las consideraciones anteriores, la Corte no deja de lado la intención del Estado de procurar por un sociedad sana, libre de vicios y 
dolencias que afecten el normal desarrollo de la misma. Sin embargo, advierte el Alto Tribunal que si bien esta actitud es lo deseable del Estado, no puede este cuando se considera que es

Respetuoso de la dignidad humana, de la autonomía personal y el libre desarrollo de la personalidad, escamotear su obligación irrenunciable de educar, y sustituir a ella la represión como forma de controlar el consumo de sustancias que se juzgan nocivas para la persona individualmente considerada y, eventualmente, para la comunidad a la que necesariamente se halla integrada (Corte Constitucional, Sentencia C-221-94, Magistrado Ponente Carlos Gaviria Díaz, Expediente N . D-429)

De todo lo anterior se vislumbra el mandato de la Corte para con el Estado de brindar desde la educación las herramientas necesarias para que cada persona en el marco de su autonomía decida cómo adelantar su proyecto de vida.

Por todo lo anterior y en el marco del enfrentamiento entre el libre desarrollo de la personalidad, el poder punitivo del Estado junto con el derecho que debe promover el Estado y el buscar las personas gozar de una buena salud expuestos en la norma relativa a la drogadicción; la Corte considera que el Estado no puede entrometerse en la esfera personal de cada una de las personas, pues el libre desarrollo de la personalidad solo es limitable por los derechos de los demás y las disposiciones normativas que son compatibles con el espíritu de la Constitución, frente a lo cual la drogadicción entendida como una afección psicofisiológica solo es nociva para la persona que la padezca y como se ha expuesto nadie está obligado a tomar un tratamiento médico si no es de su entero deseo.

\section{REFLEXIONES ENTORNO A LAS CONSIDERACIONES Y DECISIÓN DE LA CORTE CONSTITUCIONAL EN LA SENTENCIA C-221-94 PROPUESTA POR CARLOS GAVIRIA DÍAZ}

Es claro, que en el caso que se plantea ante la Corte, existe un conflicto de derechos y deberes, en el sentido de que el Estado debe promover y garantizar los derechos de sus asociados que para el planteo no es otro que el goce de un buen estado de salud ${ }^{2}$ evento que en principio cobijaría

2 Por buen estado de salud debe estarse a lo entendido y dispuesto por la OMS, es decir no tener afecciones de salud tanto física como psíquica, lo cual se expresa como un verdadero bienestar, concepto que fue aprobado por la Constitución de la OMS Organización Mundial de la Salud, aprobada en la Conferencia Internacional de Salud de 1946. 
las dependencias de las que adolece un drogadicto, pues el no consumo de la sustancia le genera afecciones en principio psíquicas y dependiendo del grado de adicción una dependencia física (tal como sucede con los adictos a la heroína u otras drogas duras).

El mencionado deber es no solo responsabilidad del Estado, sino también de la persona y así ha sido reconocido por el texto Constitucional Colombiano en el artículo 49, motivo por el cual se presenta el conflicto o tensión con el libre desarrollo de la personalidad, en el sentido de que nadie puede obligar a nadie a ser responsable de lo que no tiene convicción la cual solo le compete al titular realizar la obligación por convencimiento, o en palabras un poco más claras nadie puede obligar a otro a ejercer sus propios derechos.

Sin embargo, si la anterior interpretación fuese absoluta, se perdería de vista el deber del Estado de garantizar los derechos de sus asociados. En el caso que nos ocupa tenemos un Estado que ha decidido prestar el derecho a la salud a los drogadictos al obligarles a tomar un tratamiento médico para logar su recuperación. Lo anterior es un evento que describe un poder policivo del Estado, toda vez que esta preocupación a favor de la salud de los drogadictos ya que su afección se ha convertido en un problema para el orden jurídico, esta situación en palabras de la profesora Leticia Vita hace que la salud sea

Percibida como una cuestión privada, de la cual sólo comenzará a ocuparse el sector público desde una perspectiva de salubridad e higienismo, es decir, como una respuesta a las epidemias y demás amenazas masivas a la población económicamente activa. La salud se constituye como una cuestión de responsabilidad individual que sólo en los casos en que esa responsabilidad fallaba, se convertía en objeto de asistencia y de caridad." (2013, p.22)

Luego entonces debe entenderse que el Estado concibe a la drogadicción como una amenaza a la población económica activa, pues su mente se dispersará en los placebos que genera la droga o estupefaciente que consumen, desdibujándose entonces el marco altruista en el cual se constituye la norma que pareciere en principio estar preocupada por el problema de la drogadicción como un enemigo del bienestar de su comunidad, sino que bajo la anterior reflexión ahora se aprecia el sentido de la norma de entender a la drogadicción no como un problema de salud sino, por el contrario, como un problema para el desarrollo de la productividad y economía de la sociedad. 
Esto a todas luces desconoce uno de los fines del Estado, el cual es procurar por el bienestar de su comunidad y brindar herramientas para lograr el desarrollo de la misma. Tal vez este panorama explique por qué no solo en la Constitución Colombiana de 1.991 (y antecesoras) y el texto Constitucional de Argentina, el derecho a la salud no es reconocido como un derecho humano (fundamental), sino como un derecho cuya estirpe es de segunda categoría, adoleciendo entonces bajo esta hermenéutica de los criterios o características que propone Alexy para diferenciar a los derechos humanos, a saber: "su universalidad, su validez moral, su fundamentalidad, su prioridad y su abstracción." (Alexy, R. 200, p.4) Pues como ya se ha expuesto en la voz de la profesora Leticia Vita la salud se considera un asunto de lo privado y nada importante para la agenda pública.

Sin embargo y como ya se ha sostenido el problema de la drogadicción es un problema que afecta lo público, afectando el normal desarrollo de la sociedad así como su productividad y es por dicho motivo que no solo es tema de preocupación de los Estados en el interior de sus fronteras, sino que es un tema que ha sido seriamente, atendido por la comunidad internacional por medio de diferentes organismos en instrumentos destinados a contrarrestar esta actividad.

Ejemplo de lo anterior es la Oficina Contra las Drogas y el Delito de las Naciones Unidas, así mismo los documentos que desde este organismo se han expedido respecto a los instrumentos internacionales destinados a combatir la lucha contra las drogas. Los tres principales tratados de fiscalización internacional de drogas se apoyan mutuamente y se complementan. Un propósito importante de la Convención Única de 1961 sobre Estupefacientes y el Convenio de 1971 sobre Sustancias Psicotrópicas es codificar las medidas de control aplicables a nivel internacional con el fin de asegurar la disponibilidad de estupefacientes y sustancias psicotrópicas para fines médicos y científicos, impedir su desviación hacia canales ilícitos, incluyendo también disposiciones generales sobre el tráfico y el consumo de drogas. La Convención de las Naciones Unidas de 1988 contra el Tráfico Ilícito de Estupefacientes y Sustancias Psicotrópicas reforzó significativamente la obligación de los países a imponer sanciones penales para combatir todos los aspectos de la producción ilícita, posesión y tráfico de drogas. Pero queda sobre la mesa la duda respecto a si toda esta normativa se encuentra en el mismo sentido que ya se ha advertido respecto el verdadero motivo de las regulaciones de los Estados para combatir la drogadicción. 
Pues bien, en primer lugar no se debe perder de vista la existencia del argumento expuesto anteriormente, respecto que la preocupación por la salud de la sociedad es, en principio, una preocupación por la salubridad de esta, en el sentido de mantener en alto grado el potencial laboral y productivo de la comunidad, libre de cualquier afición que le llegase a limitar, por ello la norma no se presenta en sí como una herramienta para la atención desde la perspectiva de los humanistas del derecho a la salud, sino por el contrario una preocupación por obligar a recuperarse de su dependencia al drogadicto a fin de ser nuevamente un elemento útil para lo sociedad; el anterior planteo es recogido por el Alto Tribunal colombiano al formular las tres posibilidades hermenéuticas (anteriormente relacionadas en este texto) respecto al primer cargo formulado contra la Ley 30 de 1.986, al proponer que el propósito de la norma es evitar el dolor de la familia del drogadicto o la privación de la sociedad de un ciudadano potencialmente útil, hipótesis que como ya se expresó no prosperaron frente a la autonomía que gozan las personas en razón al libre desarrollo de la personalidad.

La Corte, a pesar de no hacer expresa referencia a la ponderación de derechos o principios, lo cierto es que las justificaciones en el marco de los argumentos de la decisión expuesta en las sentencia se sustenta en el ejercicio del método de ponderación de derechos y principios, pues el resultado ya expuesto al afirmar que el libre desarrollo de la personalidad prima sobre el "deber" del Estado de garantizar la salud de sus asociados, no es otro que las conclusiones que dicho método ofrece.

En el marco de lo anterior, la Corte y el lector de su sentencia se deben preguntar si es potestad y deber del Estado limitar el libre desarrollo de la personalidad al castigar al drogadicto con la imposición de tomar un tratamiento médico que lo lleve a recuperar su salud. Así las cosas, en este caso la Corte encontró que era innecesario e inidóneo la protección del derecho al libre desarrollo de las personas que consumen sustancias alucinógenas toda vez que el respeto por estas es uno de los pilares en los que se construye el Estado Social de Derecho, mientras que los aspectos de cierto modo se encuentran subsumidos dentro del anterior principio, por lo cual mal hace el Estado en hacer uso de la salud como un medio para justificar la imposición de penas o medidas a sus ciudadanos.

Ahora bien la imposición de un tratamiento médico como medida para asegurar el derecho a la salud de los asociados coarta la libertad del desarrollo de la personalidad, pues como ya se ha sostenido nadie está obligado a tomar un tratamiento si no lo desea, pues en la construcción de 
esta libertad se han fijado los límites de la misma en especial el de que el que afirma que con ocasión del desarrollo libre de la personalidad no se afecten los derechos otros y como es claro el drogadicto solo se afecta así mismo.

Lo anterior expone la creación de una regla (Clérico, 2010) fruto del ejercicio del método de ponderación para solución del conflicto de principios y derechos respecto al caso en comento; así las cosas, ha de prevalecer el derecho al libre desarrollo de la personalidad frente a los demás principios siempre y cuando con su ejercicio no se afecten los derechos y libertades de los demás asociados. Por tal motivo, el derecho a la salud que pretender promover el Estado con la obligatoriedad del tratamiento que solo recae sobre el drogadicto y quien solo se ve afectado en sí mismo por su adicción no justifica bajo los anteriores entendidos la limitación del libre desarrollo de la personalidad.

En armonía con lo anterior también se resalta la existencia de la tensión entre las consignas de la Ley 30 de 1.986 de corte represivo con las personas que sufren de dependencia de sustancias alucinógenas, versus los derechos a la igualdad y la dignidad humana. Así pues, frente la igualdad es necesario compartir el planteamiento de la Corte Constitucional respecto al por qué solo se castiga y obliga a tomar tratamiento a los drogadictos y no a los fumadores de nicotina y consumidores de alcohol que en todo caso siguen siendo drogas, por más aceptación social de las que estas gocen.

La discriminación se hace presente respecto al tratamiento que obligatoriamente debe recibir el drogadicto, pues una persona con recursos puede escoger libremente la entidad en donde recibirá el tratamiento al acudir al sector privado, mientras que el habitante de la calle o sin los recursos económicos suficientes deberá recibirse o será remitido a los centros estatales o de beneficencia destinados a estos menesteres. Esta consideración confirma desde la jurisprudencia el planteamiento de un sistema de salud mercantilizado, en el sentido que tendrá acceso al sistema y a mejores condiciones quien pude pagar por ello y quienes no pueden cumplir con lo anterior deberán ser cobijados por las políticas que el Estado destine para estos asuntos o las entidades de beneficencia. (Vita, 2013, p.22)

\section{CONCLUSIÓN}

De los anteriores análisis y reflexiones, salta a la vista el progresismo de Carlos Gaviria Díaz, quien como Magistrado de la Corte Constitucional 
abordó el tema relativo al consumo de sustancias alucinógenas, amparando la decisión en el respeto al principio de libre desarrollo de la personalidad, reconociendo a este como un principio rector del Estado Colombiano y expresión clara y absoluta de la autonomía de las personas, consigna que solo puede limitarse cuando con ocasión de esta libertad se afecten derechos o libertades de terceros.

Así las cosas, para el caso de los drogadictos el Estado no puede imponer la obligatoriedad de un tratamiento médico a las personas que consumen sustancias alucinógenas a modo de defensa del derecho a la salud que deben gozar todos los colombianos (es imperante recodar que en la sentencia se tipificó este tratamiento como una sanción), pues la afectación de este derecho solo se decanta al titular y no sobre terceros, tal como se dispone de la regla fruto del ejercicio de ponderación entre el libre desarrollo de la personalidad y otros derechos.

El progresismo de la Corte representado por el magistrado ponente de la sentencia se confirma con ocasión de las posiciones respecto al tema de la drogadicción, ya que es desmedido considerar al drogadicto como un delincuente y por tal motivo ser causa de la imposición de la sanción respecto del tratamiento médico, la imposición de apremios tales como multas pecuniarias y en algunos casos la privación de la libertad rompe la igualdad frente a los adictos a las sustancias socialmente aceptadas, como el alcohol y la nicotina (aceptación contenida incluso en la norma objeto de la revisión por parte de la Corte Constitucional) a quienes lógicamente su adicción no genera sanción administrativa o penal alguna. Este argumento será fundamental en el discurso de Carlos Gaviria Díaz para la eliminación del imposición del tratamiento medico para la rehabilitación, ya que como se ha sostenido en la jurisprudencia de la corporación en aras de libre desarrollo de la personalidad nadie puede ser obligado a recibir o tomar un tratamiento médico cuando no lo desea.

Ahora bien, de la preocupación altruista del Estado Colombiano de rehabilitar a sus ciudadanos con alguna afección con dependencia de sustancias alucinógenas enmarcada en la garantía del Derecho a la Salud que se predica de todos los colombianos, pareciere entonces que con la decisión de la Corte se anula esta actitud responsable del Estado. Sin embargo y con ocasión, de las reflexiones expuestas y tomando como base el texto de la profesora Leticia Vita sobre el derecho a la salud, ha sido resultado de una preocupación del Estado no procurar un bienestar para sus asociados sino a niveles de productividad de las personas a fin 
de tener seres útiles, esta posición fue incluso contemplada en las consideraciones de la Corte convirtiéndose en referente para establecer la imposibilidad de limitar el libre desarrollo de la personalidad de los drogadictos.

Lo anterior evidencia que, si bien el derecho a la salud es un logro enmarcado en los Estados sociales de reciente data, su origen y verdadero trasfondo es la concepción de una política de salubridad y foco de mercado pues como también expone la Corte en la visión de su ponente y la profesora Leticia Vita, el mercado fija los niveles de atención y calidad en servicio que reciben los asociados, situación que desvirtúa la universalidad con la que debe ser prestado el derecho a la salud.

\section{REFERENCIAS BIBLIOGRÁFICAS}

Alexy, R. (2000) La institucionalización de los derechos humanos en el Estado Constitucional Democrático. Derechos y Libertades, 5, (8). p. 124

Clérico, Laura. (2010). Hacia un modelo de la ponderación orientado por reglas para la solución de conflictos de derechos fundamentales. En: Escobar García, C. Teoría y Práctica de la justicia constitucional. Quito: Ministerio de Justicia y Derechos Humanos.

Organización Mundial de la Salud (1946). Acta Constitución de la Organización Mundial de la Salud.

Corte Constitucional de Colombia (5 de mayo de 1994). Sentencia C221. M.P: Carlos Gaviria Díaz

Organización de Naciones Unidas. Programa Drogas y Democracia de TNI. Disponible en: http://www.undrugcontrol.info/es/control-dedrogas-de-la-onu/convenciones. Consultado el 15 de octubre de 2016

Vita, Leticia. (2013) Modelos de Estado y derecho a la salud en Argentina: descubriendo los presupuestos políticos de un sistema estructuralmente desigualitario. En Clérico, L., Ronconi., L; Aldao., M Tratado de Derecho a la Salud. Buenos Aires. 